\title{
Motivos de consulta mais comuns das pessoas atendidas por uma equipe de saúde da família em Fortaleza - CE
}

The most common reasons for health encounters in a community assisted by a health team in Fortaleza, Ceará

Motivos de consulta más comunes de las personas atendidas por un equipo de salud de la familia en Fortaleza - CE

Karina de Paula Bastos Santos. Sistema Municipal Saúde Escola. Fortaleza, CE, Brasil. kkdepaula2004@ hotmail.com (Autora correspondente)

Marco Túlio Aguiar Mourão Ribeiro. Escola de Saúde Pública do Ceará. Fortaleza, CE, Brasil. marcotuliomfc@ gmail.com

\section{Resumo}

Objetivo: conhecer os principais motivos de consulta da população atendida em uma equipe de saúde do município de Fortaleza - CE. Métodos: estudo de caráter quantitativo, exploratório e transversal. Os dados foram coletados a partir dos registros dos atendimentos médicos efetuados durante o primeiro semestre de 2012, extraídos de banco de dados secundário, classificados com a CIAP-2, tabulados no Excel e analisados pelo Epi-Info. Resultados: houve um total de 1044 encontros, $68 \%$ para o sexo feminino e $32 \%$, masculino. A faixa etária predominante consistiu de pessoas entre 20-39 anos. Foram encontrados 1985 motivos de consulta. Os capítulos mais comuns foram Geral e Inespecífico, Respiratório, Digestivo, Músculo-Esquelético e Circulatório. Os 30 principais motivos de consulta corresponderam a $51,49 \%$ do total. Houve grande variedade de demandas trazidas pelas pessoas e a maioria dos motivos esteve relacionada com a demanda programada. Conclusão: a CIAP-2 possibilitou uma avaliação minuciosa da demanda. Esse estudo pode servir como instrumento para auxiliar a equipe de saúde no cuidado das pessoas, por meio de capacitação, organização da clínica e ações comunitárias e individuais para enfrentamento dos problemas.

\section{Abstract}

Objective: the aim of this study was to establish the main reasons for engaging in consultations with a health team in Fortaleza, Ceará. Methods: the data were collected from medical records covering the first semester of 2012. They were extracted from a secondary database, coded using ICPC, and then entered into an Excel spreadsheet. Results: there were 1,044 different encounters recorded, $68 \%$ with female patients and $32 \%$ with males. Most of the patients were between 20 and 39 years old. There were 1,985 reasons for encounters, the most common of which were categorized as General and Unspecified, Respiratory, Digestive, Musculoskeletal, and Cardiovascular. The main 30 reasons for encounters accounted for $51.49 \%$ of the total number. People raised a great number of demands, most of which related to the program. Conclusion: the ICPC2 program helped to precisely evaluate patients' demands. Therefore, this study showed that the program was a tool by which the health team could take care of people through capacitation, managing the clinic, and developing actions for the individuals and community to face issues that arise.
Como citar: Santos KPB, Ribeiro MTAM. Motivos de consulta mais comuns das pessoas atendidas por uma equipe de saúde da família em Fortaleza - CE. Rev Bras Med Fam Comunidade. 2015;10(37):1-11. http://dx.doi.org/10.5712/rbmfc10(37)831
Palavras-chave: Atenção Primária à Saúde Classificação de Doenças Registros Médicos

Keywords: Primary Health Care Disease Classification Medical Records
Fonte de financiamento: declaram não haver. Parecer CEP: 218.324 (Escola de Saúde Pública do Ceará - ESP/CE) aprovado em 13/03/2013 Conflito de interesses: declaram não haver. Procedência e revisão por pares: revisado por pares. Recebido em: 06/10/2013. Aprovado em: 07/05/2015. 


\section{Resumen}

Objetivo: conocer los principales motivos de consulta de la población atendida en un equipo de salud de la ciudad de Fortaleza - CE. Métodos: los datos fueron recogidos desde los registros de los atendimientos médicos realizados en el primer semestre del 2012. Ellos fueron extraídos desde un banco de datos secundario, clasificados con la CIAP-2 y analizados en Excel. Resultados: hubo una totalidad de 1044 encuentros distintos. 68\% fueron para el sexo femenino y 32\%, masculino. El grupo de edad que predomina consistió en las personas entre 20-39 años. Fueron encontrados 1985 motivos de consulta. Los capítulos más comunes fueron General e Inespecífico, Respiratorio, Digestivo, Muslo-Esquelético y Circulatorio. Los 30 principales motivos de consulta correspondieron al $51,49 \%$ de la totalidad. Existió una gran variedad de demanda traídas por las personas y la mayoría de los motivos estuvo relacionada con la demanda programada. Conclusión: la CIAP 2 posibilitó una evaluación minuciosa de la demanda. Así, ese estudio se ha tornado un instrumento para que el equipo de salud se prepare para el cuidado a esas personas, a través de capacitación, administrando mejor la clínica y desarrollando acciones comunitarias e individuales para enfrentamiento de los problemas.

\section{Palabras clave:}

Atención Primaria de Salud

Clasificación de Enfermedades Registros Médicos

\section{Introdução}

Em 1978, na Conferência de Alma Ata, firmou-se a Atenção Primária à Saúde (APS) como estratégia prioritária para ampliar o acesso à saúde, de onde partiu a meta "saúde para todos, no ano 2000". ${ }^{1}$ Como linha de frente para organização do sistema, a APS possui quatro atributos essenciais: primeiro contato, longitudinalidade, integralidade e coordenação do cuidado. ${ }^{1} \mathrm{O}$ primeiro contato está diretamente relacionado com a acessibilidade dos serviços de saúde para a população. Isso permite que os episódios de cuidado das pessoas daquele território sejam iniciados no primeiro nível de atenção, ao invés de acontecerem na atenção secundária ou terciária.

A medicina de família e comunidade (MFC), especialidade chave para a APS, surgiu como um ramo da clínica geral, em resposta ao número crescente de especialidades médicas e novas tecnologias, que fragmentaram o cuidado em saúde com abordagens que priorizavam o ponto de vista biológico. ${ }^{2} \mathrm{~A}$ MFC rompeu com essa lógica, uma vez que os seus princípios consistem em fornecer o primeiro contato com o sistema de saúde, coordenar os cuidados destinados aos pacientes, dar resolutividade aos problemas detectados e responsabilizar-se pela saúde das pessoas e da comunidade. Ela também valoriza a subjetividade na medicina, dialogando com problemas vagos e inespecíficos. Grande parte dos fatores que interferem no estado de saúde de uma pessoa - experiência com a doença, interações familiares, fatores sociais, inclusive a relação médico-paciente - são trabalhados pelos médicos de família. ${ }^{2}$ Portanto, os princípios norteadores da MFC são bastante adequados para cumprir com os atributos da atenção primária, descritos por Barbara Starfield.

O atributo da longitudinalidade corresponde ao acompanhamento contínuo, ao longo do tempo, favorecendo o conhecimento profundo dos indivíduos e o estabelecimento do vínculo. Para se cumprir com esse atributo, é essencial a existência de qualidade nos registros médicos, que, além de fornecer informações precisas sobre o estado de saúde das pessoas atendidas em determinado território, possibilitam o gerenciamento das ações dos profissionais da APS. Por isso, um bom sistema de classificação pode auxiliar na organização e análise destes dados.

Os sistemas de classificação inicialmente estavam direcionados para causas de morbidade e mortalidade. O mais conhecido deles é a Classificação Internacional de Doenças (CID). Ele é bastante útil 
para o uso de outros especialistas, uma vez que é organizado para as condições que ameaçam a saúde. ${ }^{3}$ Entretanto, ele não considera a perspectiva do paciente, seus sentimentos e expectativas, problemas sociais e familiares. Na atenção secundária e terciária esses aspectos costumam ser menos trabalhados, porém na atenção primária eles são muito importantes.

O motivo de consulta consiste na razão que leva a pessoa a procurar um serviço de saúde. A partir dele, o profissional detecta um problema e as ações a serem destinadas para a pessoa que o procura. ${ }^{4}$ Esse motivo pode ser um sintoma, uma doença, um pedido de exame ou de medicamento ou algum procedimento administrativo. A partir do desenvolvimento da Classificação de Motivos de Consulta percebeu-se que era possível e importante codificar os outros momentos da consulta - o problema detectado e o processo de cuidado, que engloba os procedimentos e condutas adotadas. Dessa forma, surgiu a Classificação Internacional de Atenção Primária (CIAP). A primeira edição foi publicada pela Organização Mundial dos Médicos de Família (WONCA) em 1987. Em 1998, a CIAP foi revisada e transformou-se em CIAP-2. Nesta versão foram incorporados os critérios de inclusão e de exclusão, para padronizar as codificações, e o mapeamento pela CID. ${ }^{5}$

O trabalho nas equipes de Saúde da Família precisa ser constantemente avaliado, uma vez que isso faz parte da gestão da clínica, com o objetivo de melhorar os indicadores de saúde e aumentar a satisfação das pessoas de determinado local. É preciso conhecer detalhadamente a demanda, os principais motivos que levam as pessoas a procurar o serviço, os diagnósticos e problemas mais comuns, assim como as intervenções realizadas. A CIAP permite que esse estudo seja feito de forma mais adequada que outros sistemas de classificação. Ainda são poucos os lugares no Brasil que começaram a utilizá-la, portanto, existem poucos estudos a esse respeito. ${ }^{3,6,7}$

A CIAP estrutura-se em episódios de cuidado. ${ }^{8}$ Estes representam os problemas de saúde. Um episódio inicia-se quando uma pessoa procura atendimento em um serviço de saúde por um determinado motivo, que pode acompanhá-la por toda a sua vida, como no caso de um indivíduo com síndrome de Down; ou pode ter início e fim, como, por exemplo, um resfriado. Um episódio de cuidado poderia originar várias consultas e em cada uma delas três elementos são essenciais: os motivos de consulta, o diagnóstico ou problema encontrado e o processo de cuidado (intervenção ou procedimento). Todos esses elementos são codificados pela CIAP.

A CIAP-2 possui uma estrutura biaxial, organizada em 17 capítulos e 7 componentes. ${ }^{8}$ O seu código é chamado de alfa-numérico, porque a primeira parte corresponde a uma letra, de determinado capítulo, e os outros dois dígitos são os números relacionados aos componentes. Os capítulos englobam os problemas de acordo com a distribuição anatômica, porém existe um capítulo geral/inespecífico, um para problemas sociais e outro para os psicológicos.

Os componentes abrangem aspectos relacionados aos sinais, sintomas, procedimentos (diagnósticos ou preventivos); medicações, tratamentos ou procedimentos terapêuticos; resultado de exames; administrativo; acompanhamento e outros motivos de consulta; diagnósticos ou doenças.

O objetivo deste estudo consistiu em identificar os principais motivos de consulta dos pacientes atendidos em uma equipe de Saúde da Família utilizando a CIAP. 


\section{Métodos}

Trata-se de um estudo de caráter quantitativo, exploratório e transversal. O banco de dados utilizado consistiu nos registros das consultas médicas presentes nos prontuários manuais de todas as pessoas atendidas pelos médicos da Equipe de Saúde da Família 31 de Março, no período de janeiro a junho de 2012. Essa equipe integra o Centro de Saúde da Família Frei Tito de Alencar, vinculado à Coordenação Regional II de Fortaleza, no Ceará, localizado na Praia do Futuro. Essa unidade é responsável por uma população de aproximadamente 20.000 pessoas, estando 3.000 sob responsabilidade da Equipe 31 de Março. O número de atendimentos dos residentes é reduzido em relação aos médicos da equipe de saúde da família que não estão em um processo formativo, para que este número esteja adequado para a discussão dos casos com os preceptores, garantindo espaço e tempo para o ensino-aprendizado.

A médica residente foi a responsável pela codificação dos registros, poucos atendimentos foram realizados por outros médicos (preceptores) presentes na equipe. Os registros de consulta foram feitos em forma de Registros Médicos Orientados por Problemas (RMOP), método bastante adequado para organização do raciocínio médico e para a investigação clínica em medicina. Foram utilizadas as notas da evolução, organizadas em SOAP (subjetivo, objetivo, avaliação e plano), as quais permitiram um acesso rápido às informações dos prontuários. Foram codificados os motivos de consulta, contidos no $\mathrm{S}$, os problemas encontrados, presentes no $A$, e as intervenções para os mesmos $(P)$. A parte objetiva $(O)$ não é codificável. Os motivos de consulta foram coletados no início da consulta, e sempre que possível eram deixados nas palavras das pessoas atendidas. Se no decorrer da consulta fossem observados outros motivos, eles poderiam ser acrescentados.

Os códigos foram organizados em uma planilha Excel e submetidos à análise estatística pelo Epi-Info. Essa codificação foi transformada em números, seguindo uma metodologia já utilizada em outros estudos. ${ }^{3,7}$ Foram utilizados, no máximo, três motivos de consulta por pessoa. Se houvesse dois motivos relacionados a um mesmo problema, os dois eram utilizados.

Foram excluídos os códigos presentes nos prontuários em que a data de nascimento ou a idade das pessoas não havia sido incluída, além dos prontuários de pessoas de outras áreas de abrangência que porventura tivessem sido atendidas pela equipe. O código utilizado para consultas de puericultura foi o A98 (Medicina Preventiva/Manutenção da Saúde), semelhante à padronização feita em outro estudo. ${ }^{3}$ Essa pesquisa não recebeu financiamento externo para a sua produção, sendo as despesas custeadas pelos autores. Não houve conflito de interesses que pudesse influenciar no resultado da pesquisa. Ela foi autorizada pela coordenação da unidade de saúde, através do Termo de Fiel Depositário, submetida ao Comitê de Ética em Pesquisa da Escola de Saúde Pública do Ceará e aprovada pelo mesmo (número do parecer 218.324, CAAE 08604613.8.0000.5037). Este artigo faz parte de um estudo maior, que contém outros objetivos e fazem parte da monografia de conclusão do Programa de Residência Médica em Medicina de Família e Comunidade.

\section{Resultados}

No período de janeiro a junho de 2012, foram atendidos 677 pacientes da área 31 de Março. Destes, 446 realizaram atendimento médico mais de uma vez, resultando em um total de 1044 consultas e uma média de 1,5 encontros por pessoa. Esse número de consultas foi o total de atendimentos disponibilizados 
para a população desta área de abrangência, o que corresponde a uma média de nove consultas por dia no período realizado. Houve um grande número de consultas para o mesmo paciente, devido à quantidade de gestantes, hipertensos e diabéticos, cujo acompanhamento necessitava de vários encontros. $\mathrm{O}$ sexo predominante correspondeu ao feminino, com um total de $68 \%$ das consultas, enquanto o masculino obteve $32 \%$. A faixa etária com maior número de atendimentos correspondeu às pessoas com idade de 20 a 39 anos, como pode ser visto no Gráfico 1.

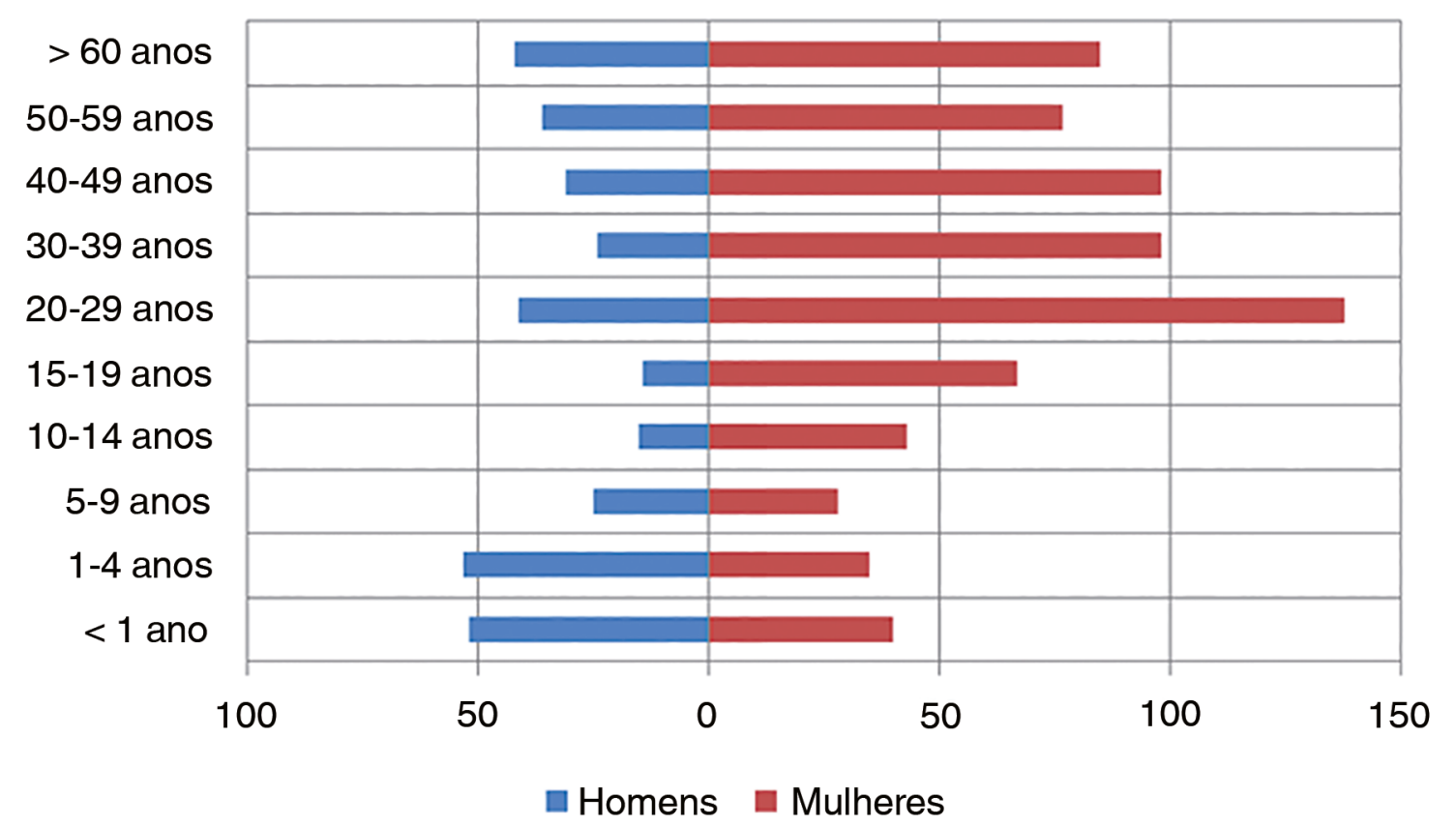

Gráfico 1. DDistribuição dos encontros por faixa etária e sexo em uma equipe de saúde da família de Fortaleza, 2012

Esse estudo encontrou 1985 motivos de consulta. A frequência dos mesmos, organizada por capítulo e por códigos específicos, pode ser vista na Tabela 1. Os mais prevalentes corresponderam aos códigos do capítulo Geral e Inespecífico, Respiratório, Digestivo, Musculoesquelético e Circulatório.

Na Tabela 2, observa-se diferença entre as frequências dos motivos por capítulos de acordo com o sexo.

Os 30 principais motivos de consulta corresponderam a $51,49 \%$ do total, como pode ser visto na Tabela 3. Na faixa etária dos 15 aos 19 anos, o motivo Gravidez teve maior prevalência.

A distribuição dos motivos de consulta de acordo com os componentes da CIAP pode ser vista no Gráfico 2. A maioria deles $(51,1 \%)$ pertenciam ao componente 1, o qual engloba os sintomas. Foi possível perceber que $24,8 \%$ eram diagnósticos definitivos (componente 7 ) trazidos pelas pessoas, aceitos nos motivos de consulta, mesmo que na avaliação do profissional a doença em questão não fosse confirmada.

\section{Discussão}

Aanálise dos principais motivos contribui para a capacitação dos profissionais da APS e entendimento do perfil dos pacientes que buscam a unidade. A distribuição por sexo foi similar à encontrada em outros estudos de demanda brasileiros, corroborando o fato de as consultas na atenção primária terem uma 
Tabela 1. Prevalência dos motivos de consulta por capítulos da CIAP em uma equipe de saúde da família de Fortaleza, 2012.

\begin{tabular}{lcc}
\hline Capítulos & Frequência & Percentual \\
\hline A - Geral e Inespecífico & 243 & $12,24 \%$ \\
R - Respiratório & 228 & $11,49 \%$ \\
D - Digestivo & 221 & $11,13 \%$ \\
L - Musculoesquelético & 204 & $10,28 \%$ \\
K - Circulatório & 187 & $9,42 \%$ \\
S - Pele & 182 & $9,17 \%$ \\
W - Gravidez, parto e planejamento familiar & 135 & $6,8 \%$ \\
X - Genital feminino & 133 & $6,7 \%$ \\
T - Endócrino/Metabólico e Nutricional & 132 & $6,65 \%$ \\
N - Neurológico & 120 & $6,05 \%$ \\
P - Psicológico & 72 & $3,63 \%$ \\
U - Urinário & 44 & $2,22 \%$ \\
F - Olho & 33 & $1,66 \%$ \\
H - Ouvido & 31 & $1,56 \%$ \\
Y - Genital Masculino & 31 & $0,55 \%$ \\
B - Sangue, Sistema Hematopoiético, Linfático e Baço & 11 & $0,45 \%$ \\
Total & 9 & $100 \%$ \\
\hline
\end{tabular}

Tabela 2. Prevalência dos Motivos de Consulta por capítulo de acordo com o sexo em uma equipe de saúde da família de Fortaleza, 2012.

\begin{tabular}{|c|c|c|c|c|c|}
\hline \multicolumn{3}{|c|}{ Mulheres } & \multicolumn{3}{|c|}{ Homens } \\
\hline Capítulos & $\#$ & $\%$ & Capítulos & $\#$ & $\%$ \\
\hline D - Digestivo & 149 & $10,7 \%$ & A - Geral e Inespecífico & 109 & $18,6 \%$ \\
\hline L - Musculoesquelético & 148 & $10,6 \%$ & R - Respiratório & 96 & $16,3 \%$ \\
\hline W - Gravidez/Planej. & 135 & $9,7 \%$ & S - Pele & 74 & $12,6 \%$ \\
\hline A - Geral e Inespecífico & 134 & $9,6 \%$ & D - Digestivo & 72 & $12,3 \%$ \\
\hline R - Respiratório & 132 & $9,4 \%$ & K - Circulatório & 63 & $10,7 \%$ \\
\hline X - Genital Feminino & 132 & $9,4 \%$ & L - Musculoesquelético & 56 & $9,5 \%$ \\
\hline K - Circulatório & 124 & $8,9 \%$ & $\mathrm{~T}$ - Endocrino & 37 & $6,3 \%$ \\
\hline S - Pele & 108 & $7,7 \%$ & $\mathrm{~N}$ - Neurológico & 27 & $4,6 \%$ \\
\hline T - Endócrino & 95 & $6,8 \%$ & H - Ouvido & 14 & $2,4 \%$ \\
\hline N - Neurológico & 93 & $6,6 \%$ & Y - Genital Masculino & 11 & $1,9 \%$ \\
\hline P - Psicológico & 63 & $4,6 \%$ & P - Psicológico & 9 & $1,5 \%$ \\
\hline
\end{tabular}

predominância do sexo feminino. 3,7,9 Isso pode estar relacionado com questões culturais e históricas. Esse estudo também demonstrou que os homens procuraram mais a unidade devido a queixas agudas. Um estudo de demanda realizado em quatro países demonstrou divisão por sexos igualitária, exceto nos dados dos Estados Unidos, em que o sexo feminino ocupou $59 \%$ das consultas. ${ }^{10}$ Resultados semelhantes a esse estudo foram encontrados em duas cidades da China. ${ }^{11}$

Adolescentes demandaram poucas consultas médicas, quando comparados com as outras faixas etárias, semelhante ao encontrado em outros estudos. ${ }^{3,9-11}$ Geralmente, essa faixa etária adoece pouco, mas a equipe de saúde precisa saber se esse é o motivo pelo qual eles não procuram a unidade ou se existe alguma dificuldade no acesso. A maioria dos que procuraram foi devido a questões relacionadas com a gravidez e puerpério. Isso demonstra que esse problema é muito importante na adolescência nesta 
Tabela 3. Motivos de consulta mais frequentes em uma equipe de saúde da família de Fortaleza, 2012.

\begin{tabular}{|c|c|c|c|}
\hline Motivo de Consulta & Frequência & Porcentagem & Porcentagem acumulada \\
\hline W78 - Gravidez & 85 & $4,27 \%$ & $4,27 \%$ \\
\hline N01 - Cefaleia & 78 & $3,92 \%$ & $8,19 \%$ \\
\hline A98 - Medicina Preventiva/Manutenção da Saúde & 78 & $3,92 \%$ & $12,11 \%$ \\
\hline K86 - Hipertensão sem complicações & 75 & $3,77 \%$ & $15,88 \%$ \\
\hline S06 - Erupção cutânea localizada & 61 & $3,06 \%$ & $18,94 \%$ \\
\hline T90 - Diabetes não insulino dependente & 59 & $2,96 \%$ & $21,9 \%$ \\
\hline R05 - Tosse & 58 & $2,91 \%$ & $24,81 \%$ \\
\hline A03 - Febre & 53 & $2,66 \%$ & $27,47 \%$ \\
\hline R80 - Gripe & 49 & $2,46 \%$ & $29,93 \%$ \\
\hline D50 - Medicação/prescrição/renovação/injeção (capítulo Digestivo) & 37 & $1,86 \%$ & $31,79 \%$ \\
\hline K50 - Medicação/prescrição/renovação/injeção (capitulo Circulatório) & 35 & $1,76 \%$ & $33,55 \%$ \\
\hline R21 - Sinais/sintomas da garganta & 29 & $1,46 \%$ & $35,01 \%$ \\
\hline L03 - Sinais/sintomas da região lombar & 26 & $1,31 \%$ & $36,32 \%$ \\
\hline D11 - Diarreia & 25 & $1,26 \%$ & $37,58 \%$ \\
\hline K60 - Resultado de Análises/Procedimentos (capítulo Circulatório) & 24 & $1,21 \%$ & $38,79 \%$ \\
\hline L14 - Sinais/sintomas da coxa/perna & 21 & $1,05 \%$ & $39,84 \%$ \\
\hline A60 - Resultado de Análises/Procedimentos (capitulo Geral e Inespecífico) & 20 & $1 \%$ & $40,84 \%$ \\
\hline X14 - Secreção Vaginal & 19 & $0,95 \%$ & $41,79 \%$ \\
\hline X60 - Resultado de Análises/Procedimentos (Capítulo Genital Feminino) & 19 & $0,95 \%$ & $42,74 \%$ \\
\hline S08 - Alterações da cor da pele & 18 & $0,90 \%$ & $43,64 \%$ \\
\hline D01 - Dor abdominal generalizada/cólicas & 18 & $0,90 \%$ & $44,54 \%$ \\
\hline D02 - Dores abdominais, epigástricas & 18 & $0,90 \%$ & $45,44 \%$ \\
\hline L15 - Sinais/sintomas do joelho & 17 & $0,85 \%$ & $46,29 \%$ \\
\hline X05 - Menstruação escassa/ausente & 16 & $0,80 \%$ & $47,09 \%$ \\
\hline P64 - Episódio/problema iniciado pelo prestador (capítulo Psicológico) & 15 & $0,75 \%$ & $47,84 \%$ \\
\hline $\begin{array}{l}\text { T50 - Medicação/prescrição/renovação/injeção (capitulo Endócrino/ } \\
\text { Metabólico e Nutricional) }\end{array}$ & 15 & $0,75 \%$ & $48,59 \%$ \\
\hline L17 - Sinais/sintomas do pé/dedos dos pés & 15 & $0,75 \%$ & $49,34 \%$ \\
\hline D10 - Vômitos & 15 & $0,75 \%$ & $50,09 \%$ \\
\hline X17 - Sinais/sintomas da pélvis feminina & 14 & $0,70 \%$ & $50,79 \%$ \\
\hline R02 - Dificuldade respiratória, dispneia & 14 & $0,70 \%$ & $51,49 \%$ \\
\hline
\end{tabular}

comunidade, indicando que as atividades de planejamento reprodutivo realizadas pela equipe precisam ser mais efetivas, no intuito de reverter esse quadro. ${ }^{12}$ Este capítulo também foi bastante frequente nesta faixa etária em um estudo da África do Sul, porém as adolescentes procuravam as unidades para consultas de contracepção. ${ }^{13} \mathrm{O}$ capítulo Geral é o que engloba grande parte dos motivos de consulta na maioria dos estudos. ${ }^{3,6,7,10,11} \mathrm{Em}$ um estudo piloto brasileiro, os capítulos respiratório e digestivo também estiveram entre os primeiros. ${ }^{6} \mathrm{O}$ capítulo Genital Masculino e o relacionado com o sistema hematopoiético foram os que tiveram as menores frequências, semelhantes às encontradas nos outros estudos. ${ }^{3,7,10,11,14} \mathrm{~A}$ baixa prevalência do capítulo Psicológico pode ser um reflexo da dificuldade que os médicos de família possuem na abordagem de queixas psicológicas, conforme apontado em um estudo europeu. ${ }^{15}$

A Tabela 4 demonstra os sete principais motivos de consulta no estudo em questão e em outros estudos. Dos mais frequentes, três eram relacionados com consultas de ação programática, o que sugere 


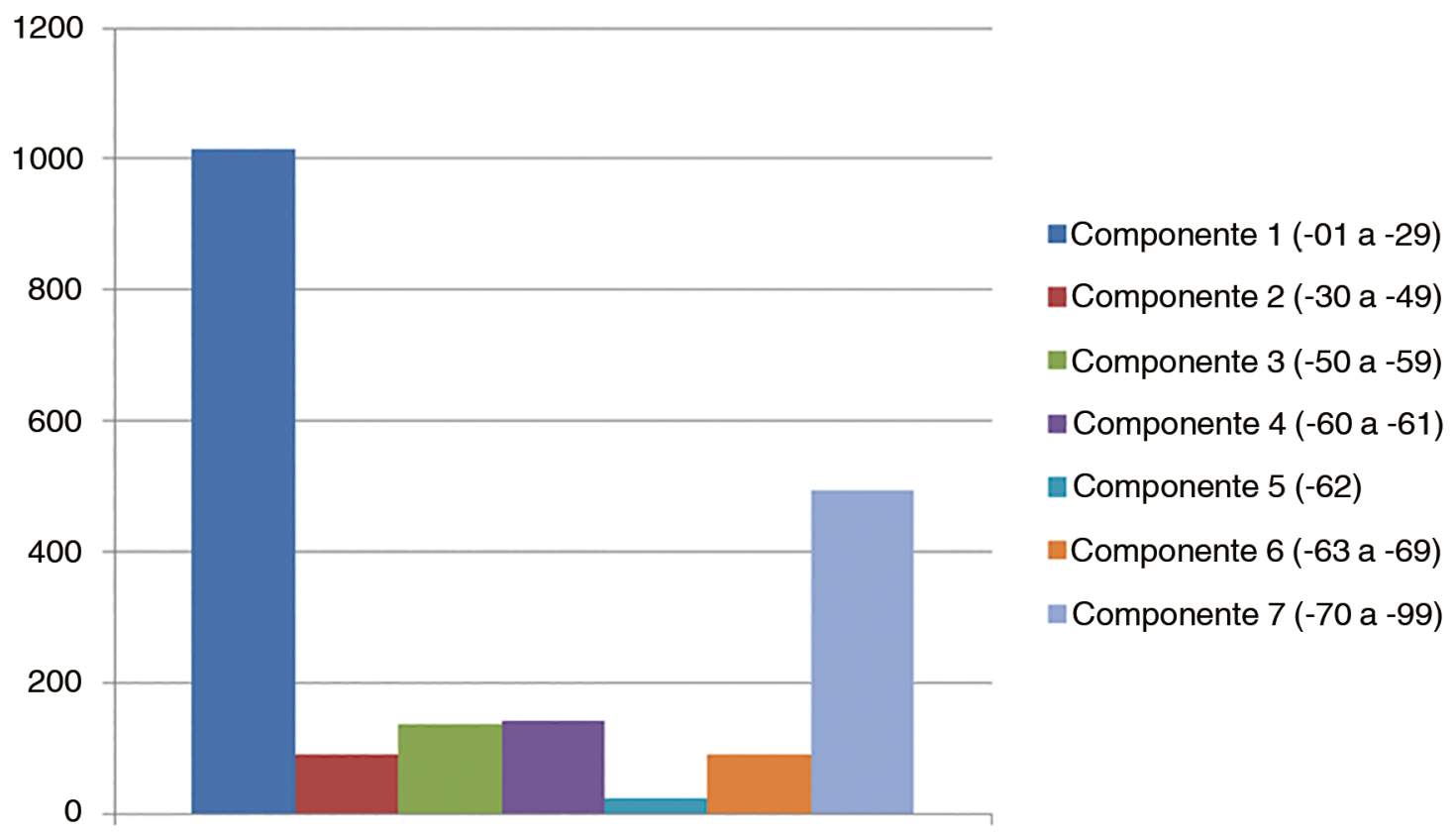

Gráfico 2. Natureza dos motivos de consulta em uma equipe de saúde da família de Fortaleza, 2012.

que a agenda dos profissionais pode estar organizada de acordo com os programas do Ministério da Saúde. O estudo de Betim obteve uma maior frequência de cefaleia, febre e tosse como motivos relacionados aos sintomas. ${ }^{7}$ Essa diferença pode ser atribuída ao fato de esse estudo analisar apenas a demanda espontânea, excluindo a programada. No estudo realizado em Florianópolis, o motivo de consulta mais comum consistiu em Medicina Preventiva/Manutenção de Saúde, seguido de tosse, pedido de medicação do aparelho circulatório, febre e gravidez. ${ }^{3}$

Tabela 4. Motivos de consulta mais frequentes em alguns estudos.

\begin{tabular}{|c|c|c|c|c|c|c|}
\hline $\begin{array}{l}\text { Ordem de } \\
\text { frequência }\end{array}$ & $\begin{array}{c}\text { Estudo em } \\
\text { questão (tabela 3) }\end{array}$ & Florianópolis ${ }^{3}$ & Betim $^{7}$ & China $^{11}$ & Holanda ${ }^{4}$ & África $^{13}$ \\
\hline 1 & Gravidez & $\begin{array}{l}\text { Medicina Preventiva/ } \\
\text { manutenção da } \\
\text { saúde }\end{array}$ & $\begin{array}{l}\text { Resultado de análi- } \\
\text { ses/procedimentos }\end{array}$ & Tosse & $\begin{array}{l}\text { Exames } \\
\text { especial-circulatório }\end{array}$ & Tosse \\
\hline 2 & Cefaleia & Tosse & $\begin{array}{l}\text { Medicação/Pres- } \\
\text { crição/renovação/ } \\
\text { injeção-geral }\end{array}$ & Vertigens/tonturas & Tosse & Cefaleia \\
\hline 3 & $\begin{array}{l}\text { Medicina Preventiva/ } \\
\text { manutenção da } \\
\text { saúde }\end{array}$ & $\begin{array}{l}\text { Medicação/Pres- } \\
\text { crição/renovação/ } \\
\text { injeção-circulatório }\end{array}$ & Cefaleia & Febre & $\begin{array}{l}\text { Hipertensão sem } \\
\text { complicações }\end{array}$ & Febre \\
\hline 4 & $\begin{array}{l}\text { Hipertensão sem } \\
\text { complicações }\end{array}$ & Febre & Febre & $\begin{array}{l}\text { Sinais/sintomas da } \\
\text { garganta }\end{array}$ & Contracepção & $\begin{array}{l}\text { Sinais e sintomas } \\
\text { nasais }\end{array}$ \\
\hline 5 & $\begin{array}{l}\text { Erupção cutânea } \\
\text { localizada }\end{array}$ & Gravidez & Tosse & Cefaleia & $\begin{array}{l}\text { Sensação de Ansie- } \\
\text { dade/nervosismo }\end{array}$ & $\begin{array}{l}\text { Sinais e sintomas } \\
\text { da garganta }\end{array}$ \\
\hline 6 & $\begin{array}{l}\text { Diabetes não } \\
\text { insulinodependente }\end{array}$ & Cefaleia & $\begin{array}{l}\text { Dor generalizada, } \\
\text { múltipla }\end{array}$ & Palpitações & $\begin{array}{l}\text { Medicação/Pres- } \\
\text { crição/renovação/ } \\
\text { injeção-circulatório }\end{array}$ & $\begin{array}{l}\text { Sinais e sintomas } \\
\text { da região lombar } \\
\text { e dorsal }\end{array}$ \\
\hline 7 & Tosse & $\begin{array}{l}\text { Hipertensão sem } \\
\text { complicações }\end{array}$ & $\begin{array}{l}\text { Medicação/Pres- } \\
\text { crição/renovação/ } \\
\text { injeção-circulatório }\end{array}$ & $\begin{array}{l}\text { Sensação de pres- } \\
\text { são/aperto no peito } \\
\text { atribuída ao coração }\end{array}$ & $\begin{array}{l}\text { Medicação/Pres- } \\
\text { crição/renovação/ } \\
\text { injeção-psicológico }\end{array}$ & Dor generalizada \\
\hline
\end{tabular}


Apenas dez desses motivos coincidiram com os mais frequentes em cenários internacionais, conforme demonstra um estudo realizado a partir da coleta de dados na Holanda, Japão, Estados Unidos e Polônia. ${ }^{10}$ Nesse estudo, os autores coletaram os motivos de consulta, diagnósticos e intervenções no cenário de atenção primária. Os capítulos mais frequentes foram semelhantes - digestivo, circulatório, musculoesquelético, respiratório e pele. Entretanto, consideraram apenas os motivos do componente 1 da CIAP. As diferenças encontradas podem ter uma relação com as particularidades de cada local. No estudo de demanda realizado em duas cidades da China, os motivos mais comuns consistiram em hipertensão sem complicações e diabetes não insulino-dependente, no grupo de doenças, e cefaleia e lombalgia como sintomas, os quais também foram encontrados neste estudo. ${ }^{11}$

Quanto à natureza dos motivos, a quantidade de diagnósticos definitivos demonstra que algumas pessoas já procuraram o serviço com uma patologia, pode ser por causa de uma doença crônica ou devido à opinião deles sobre seus sintomas. Esses dados foram semelhantes aos encontrados no estudo piloto da CIAP realizado na Holanda, ${ }^{4}$ porém diferentes daqueles encontrados no estudo pioneiro da CIAP no Brasil, no qual $70 \%$ dos motivos pertenciam ao componente 1 , seguido do componente $2(9,8 \%)$ e componente 7 $(7,3 \%) .{ }^{6}$ Entretanto, esse estudo utilizou como fonte de dados não apenas registros da atenção primária, mas também de outros níveis da atenção.

A quantidade de consultas iniciadas pelo prestador também foi pequena, demonstrando que, na maioria das vezes, a pessoa trazia seus motivos de forma espontânea. Provavelmente, a agenda priorizada era a do paciente e não a do médico. Além disso, foi escasso o número de motivos que consistiam em pedido de encaminhamento para outro especialista, o que pode mostrar que a equipe consegue atender às necessidades da população ou então que a população não possui a tendência de solicitar de imediato um encaminhamento para outro especialista. Esse achado foi bastante similar a um outro estudo de demanda realizado anteriormente em Fortaleza. ${ }^{9}$

Não foi possível fazer a análise por episódio de cuidado, uma vez que para isso é necessário um prontuário eletrônico para correlacionar os motivos de consulta e diagnósticos referentes a um mesmo problema. Além disso, a análise foi efetuada com os atendimentos realizados em um semestre, não sendo possível afirmar se os mesmos são representativos do padrão do ano inteiro. A não utilização dos códigos relacionados ao capítulo social pode ter prejudicado este estudo, uma vez que a população atendida era bastante vulnerável a problemas sociais.

A CIAP tem limitações. Alguns aspectos da consulta perdem-se pelo fato de não serem codificáveis. Além disso, a classificação, em alguns momentos, simplifica a complexidade envolvida no trabalho do médico de família e comunidade. Essa ferramenta depende diretamente da capacitação que o médico possui para realizar a codificação, das suas habilidades de comunicação e do relacionamento e vínculo que ele tem com seus pacientes, para descobrir o real motivo da vinda deles ao consultório.

É importante ressaltar a baixa quantidade de consultas ofertadas para a população da qual a equipe é responsável, uma vez que houve uma média de 0,7 atendimentos por pessoa/ano, metade da média nacional, e bem abaixo do valor mais sugerido internacionalmente - 3 a 4 consultas/pessoa/ano. ${ }^{16}$ Este fato poderia ser explicado pelo número médio de consultas realizadas pela médica residente, que para garantir o cenário adequado para o seu aprendizado, tem uma agenda reduzida. De toda forma, este achado pode sugerir que esta pode ser uma ferramenta importante para a discussão do processo de trabalho da equipe e do modelo de preceptoria em que a residência está estruturada. 
Esse estudo demonstrou a grande variedade de condições que se apresentam na atenção primária. A CIAP demonstrou ser um instrumento bastante útil para conhecer a demanda da população atendida, tornando possível o entendimento das etapas do processo diagnóstico, além de ser uma nova ferramenta para trabalhar a epidemiologia da Medicina de Família e Comunidade. Quando existem equipes de saúde que realizam esse tipo de estudo do seu território de abrangência, a comunidade terá profissionais com um conhecimento profundo sobre os problemas existentes na mesma. O desafio consiste em saber se os egressos das residências em Medicina de Família e Comunidade e das residências multiprofissionais em Saúde da Família adquiriram a formação adequada para lidar com esses problemas; para responder essa questão seriam necessários outros estudos.

\section{Conclusão}

Os dados encontrados no presente estudo foram semelhantes aos de outros estudos brasileiros, quanto ao perfil da população atendida e às demandas. Em relação aos estudos internacionais, houve semelhança dos dados em alguns capítulos para os motivos de consulta. Ao conhecer as condições mais comuns da prática, cabe ao médico preparar-se para as mesmas, de forma individual, com as capacitações e educação permanente e em conjunto com os outros profissionais. Dessa forma, é possível trabalhar com a gestão da clínica nas unidades para lidar de forma resolutiva com os problemas encontrados.

\section{Referências}

1. Starfield B. Atenção Primária: equilíbrio entre necessidades de saúde, serviços e tecnologia. Brasília:UNESCO, Ministério da Saúde; 2002. 726p.

2. McWhinney IR, Freeman T. Manual de medicina de família e comunidade. 3a ed. Porto Alegre: Artmed; 2010. p.472.

3. Gusso GDF. Diagnóstico de demanda em Florianópolis utilizando a classificação internacional de atenção primária. 2a ed (CIAP 2) [Tese de doutorado]. São Paulo: Universidade de São Paulo; 2009. 212p.

4. Lamberts $\mathrm{H}$, Meads $\mathrm{S}$, Wood M. Classification of reasons why persons seek primary care: pilot study of a new system. Public Health Rep. 1984;99(6):597-605.

5. Soler JK, Okkes I, Wood M, Lamberts H. The coming of age of ICPC: celebrating the 21st birthday of the International Classification of Primary Care. Fam Pract. 2008;25(4):312-7. DOI: http://dx.doi.org/10.1093/fampra/cmn028

6. Lebrão ML. Classificação internacional de motivos da consulta para atenção primária: testes em algumas áreas brasileiras. Rev Saúde Pública. 1985;19(1):69-78. DOI: http://dx.doi.org/10.1590/S0034-89101985000100008

7. Landsberg GAP, Savassi LCM, Sousa AB, Freitas JMR, Nascimento JLS, Azagra R. Análise de demanda de Medicina de Família no Brasil utilizando a Classificação Internacional de Atenção Primária. Ciênc Saúde Coletiva. 2012;17(11):302536. DOI: http://dx.doi.org/10.1590/S1413-81232012001100019

8. Gusso GDF. Classificação Internacional de Atenção Primária (CIAP 2) [Tese de Doutorado]. São Paulo: Faculdade de Medicina da Universidade de São Paulo; 2009.

9. Pimentel IRS, Coelho BC, Lima JC, Ribeiro FG, Sampaio FPC, Pinheiro RP et al. Caracterização da demanda em uma Unidade de Saúde da Família. Rev Bras Med Fam. 2011;6(20):175-81. DOI: http://dx.doi.org/10.5712/rbmfc6(20)95

10. Okkes IM, Polderman GO, Fryer GE, Yamada T, Bujak M, Oskam SK, et al. The role of family practice in different health care systems: a comparison of reasons for encounter, diagnoses, and interventions in primary care populations in the Netherlands, Japan, Poland, and the United States. J Fam Pract. 2002;51(1):72-3. http://www.jfponline.com/pages. asp?aid=1085.

11. Wun Y, Lu X, Liang W, Dickinson J. The work by the developing primary care team in China: a survey in two cities. Fam Pract. 2000;17(1):10-5. DOI: http://dx.doi.org/10.1093/fampra/17.1.10 
12. Brasil. Ministério da Saúde. Secretaria de Atenção à Saúde, Departamento de Atenção Básica. Saúde Sexual e Saúde Reprodutiva. Cadernos de Atenção Básica número 26. Brasília; 2010.

13. Mash B, Fairall L, Adejayan O, Ikpefan O, Kumari J, Mathee S, et al. A morbidity survey of South African primary care. PLoS One. 2012;7(3):e32358. DOI: http://dx.doi.org/10.1371/journal.pone.0032358

14. Moth G, Olesen F, Vedsted P. Reasons for encounter and disease patterns in Danish primary care: changes over 16 years. Scand J Prim Health Care. 2012;30(2):70-5. DOI: http://dx.doi.org/10.3109/02813432.2012.679230

15. Rosendal M, Vedsted P, Christensen KS, Moth G. Psychological and social problems in primary care patients - general practitioners' assessment and classification. Scand J Prim Health Care. 2013;31(1):43-9. DOI: http://dx.doi.org/10.3109/ 02813432.2012 .751688

16. Gusso GDF, Poli Neto P. Gestão da Clínica. In: Gusso GDP, Lopes JMC, eds. Tratado de medicina de família e comunidade: princípios, formação e prática. Porto Alegre: Artmed; 2012. p.159-66. 